\title{
The older traveller
}

\section{Marcin Woś ${ }^{1}$, Krzysztof Korzeniewski $^{2}$}

Students' Scientific Circle of Travel Medicine, Medical University of Warsaw, Poland

${ }^{2}$ Department of Epidemiology and Tropical Medicine; Military Institute of Medicine, Warsaw, Poland

\begin{abstract}
All over the world there has been a sudden increase in the number of international travels, mostly for touristic purposes. According to the World Tourism Organisation, the number of international journeys exceeded 1.323 billion in 2017 and it continues to grow. Of the growing number of travellers more and more are the elderly (> 65 years), this fact can be attributed to longer life expectancy and a better quality of life, especially in the developed countries. The article lists the main destinations chosen by senior tourists and their reasons for travel, it also discusses physiological changes in organs and systems affecting the elderly, which are the result of travelling across time zones and to areas with different environment. The article looks at various groups of patients affected by chronic diseases and examines health-related consequences of travel, including the most common complications. The general health prevention measures, with emphasis on vaccinations and antimalarial chemoprophylaxis, have also been discussed.
\end{abstract}

(Int Marit Health 2018; 69, 4: 285-296)

\section{Key words: older traveller, travel-related disease, health prevention}

\section{INTRODUCTION}

The number of travelling older adults (> 65 years) is increasing every year, in keeping with growing numbers of travellers at all ages. According to the World Tourism Organisation [1], the number of international tourist arrivals reached 1.323 billion in 2017 and continues to grow. It is widely known that the population worldwide is getting older. Indeed, $8.7 \%$ of the world population is 65 and above [2]. One of the reasons for this is that in high-income countries live expectancy has been steadily increasing for decades [3]. According to European Commission statistics, live expectancy at birth in the European Union is estimated at 83.6 years for women and 78.2 for men [4]. In the study performed by Kontis et al. [3], projections show continued increases in longevity in 35 industrialised countries. The number of elderly travellers is constantly growing not only because of the increased live expectancy, but also due to the improvement of the quality of life and of medical technology, significantly higher income, relatively few obligations and a longer time spent in retirement [5-7]. Normal aging is associated with physiological changes affecting all systems, especially the cardiovascular, respiratory, gastrointestinal, dermal, renal, endocrine, and musculoskeletal [8]. Therefore, elderly people are at an increased risk of negative effects associated with their underlying medical conditions and medications they receive [9]. Concomitantly, physiological changes put older travellers at a much higher risk of travel-associated infections and complications compared with younger patients [10]. For this reason, the effects of aging and physiological changes associated with this process are particularly important while discussing travel-related diseases. As international travels have become increasingly available to larger groups of travellers, elderly individuals now represent an increasing proportion of patients with travel-associated diseases [11]. Furthermore, as the number of elderly travellers is constantly growing, there may be an increase in the number of elderly patients who seek pre-travel counselling [10].

\section{EPIDEMIOLOGY}

It is estimated that $15-30 \%$ of the total number of international travellers are people aged 60 years or more [11]. According to European Commission statistics, 48.8\% of European Union citizens aged 65 and over took part in international and domestic travels in 2015. The number 
of elderly travellers differed between European countries and ranged between $83.7 \%$ in Denmark and only $11.0 \%$ in Bulgaria [12]. In the study performed by Gautret et al. [11], $49.1 \%$ of tourists aged 60 years and over were from Europe, $36.4 \%$ from North America and $6.7 \%$ from Asia. Destinations chosen by senior travellers are also important; travel to developing countries has become increasingly popular. In the study by Gautret et al. [11], 47.1\% of elderly travellers have chosen Asia as their travel destination, $21.9 \%$ went to Africa, $16.5 \%$ to Latin America and only $7.7 \%$ to Europe and $2.4 \%$ to North America. In another study performed by Alon et al. [13], 53.1\% of elderly travellers went to East Asia, $30.3 \%$ to South America and $14.6 \%$ to Africa. In comparison, in the Swiss study [5] the main destination were the tropical countries in South America. The other important issues to consider are the travel mode and conditions. The majority of senior travellers opted for organised tours (61.4\%); however, $10.4 \%$ of them backpacked [13]. According to Jaeger et al. [5], 8.1\% travelled on a low budget. Regardless of age, visiting South-East Asia and travel under basic conditions is associated with a higher risk of acquiring travel-related diseases [13]. What is more, there is a relationship between nationality and the travel destination choice. For elderly European travellers security is the priority, for Americans - it is comfort, for Canadians - entertainment and for Japanese seniors - health and safety [6]. The median travel duration is generally shorter in the elderly compared to younger travellers [11]. The group of older patients complied better with malaria chemoprophylaxis and dietary restrictions. Drinking and eating habits were also different between younger and older tourists. Only $16.2 \%$ of elderly travellers ate food from street vendors, compared to $37.9 \%$ of the younger travellers. No more than $8 \%$ of elderly travellers admitted drinking tap water, while among the younger travellers the figures were $35 \%$ [13]. In the study performed by Gautret et al. [11], the main reason for journey for $66.3 \%$ of 7034 travellers aged 60 years and older was tourism. Other common reasons for travel in this group were: business (16.7\%), missionary, volunteer, research or aid work (8.5\%), and visiting friends and relatives (8.0\%). In the study carried out by Jaeger et al. [5], the main reasons included: tourism (87\%), visiting friends and relatives (7.3\%), and business (3.1\%). The most popular activities among senior travellers during their trips are: going on city tours, visiting historic places, eating out and shopping, while they are generally not interested in water sports, sunbathing, camping or hiking [6]. Some of the elderly travellers perceive retirement as an opportunity to travel without any pressure. They can spend more time at a given destination and get to know it better [7]. Contrary to other age groups, $48 \%$ of elderly people indicated health as one of the main reasons not to travel [14]. According to Sundling et al. [15], health was the main obstacle to railway travel only for patients with low functional ability, whereas older travellers with high functional ability put costs and poor punctuality as the main obstacle to travelling more often. On the other hand, some elderly people who used to travel a lot as part of their job perceive retirement as an opportunity to travel less [7].

\section{AGE-RELATED PHYSIOLOGICAL CHANGES AND THEIR CONSEQUENCES}

\section{CARDIOVASCULAR SYSTEM}

Age is the greatest risk factor for cardiovascular disease [16]. There is a general tendency for the cardiac output to decline and systolic hypertension is much more likely to occur in this group of patients. Another problem of geriatric patients is arteriosclerosis. Stiffening, fibrosis and incremental accumulation of phosphate and calcium occur during the aging process $[8,9]$. Arteriosclerosis is the leading cause of coronary artery disease (CAD) [17], whereas age and hypertension are significant risk factors $[8,17]$. Cardiovascular events are a common cause of in-flight medical emergencies, with exacerbations in CAD being one of the main incidences. Some diseases, e.g. mild and treated appropriately stable chronic angina and stable heart failure or controlled systemic hypertension are not contraindications to flying. What is more, it is even possible to fly 1 week after uncomplicated myocardial infarction or 5 days after uncomplicated percutaneous coronary intervention. However, patients with severe heart failure (New York Heart Association IV) or those with unstable angina are not recommended to travel by air. Individuals with moderate to severe systolic pulmonary hypertension (higher than $60)$ may need supplementary oxygen. Another important issue is venous thromboembolism. The hazard is higher in patients with pre-existing risk factors, e.g. older age, malignancy, obesity, recent surgeries, inherited or acquired thrombophilia, polycythaemia or oestrogen therapy $[10,18]$. As pointed out by Lee et al. [10] patients at the highest risk of venous thromboembolism may benefit from well fitted medical compression stockings and subcutaneous enoxaparin administered before and 1 day after air travel if the journey lasts more than 3 hours.

\section{RESPIRATORY SYSTEM}

Aging-related changes in the respiratory system, including a decrease in vital capacity, an increase in residual volume, a reduction amount of protective mucous, impaired cough reflex, insufficient production of Ig A may all have a negative effect on elderly travellers $[8,9]$. According to Guatret et al. [11], 14.6\% of travellers aged 60 and over had a respiratory tract infection, ranked as the second most common complaint in this group of patients. Even 
though elderly travellers present less frequently with upper respiratory tract infections, they are at a higher risk of developing lower respiratory tract infections, e.g. pneumonia and bronchitis. Therefore, preventive measures against respiratory infections are particularly important for older adults $[11,13]$. Another problem is hypobaric hypoxemia induced by high altitude and a poorer ventilatory response to hypoxia compared with younger patients, which may lead to exacerbation of underlying pulmonary diseases $[10,19]$. Lee et al. [10] suggest that for this reason geriatric patients with certain chronic (e.g. chronic obstructive pulmonary disease) or acute (e.g. pneumonia) medical problems should contact a clinician prior to travel to assess the risk of exacerbation. It is also essential to contact the airline before travel in the event that supplemental oxygen is required. In addition, older age may be a risk factor for developing high-altitude illness.

\section{GASTROINTESTINAL SYSTEM}

Changes in the gastrointestinal system occur with advancing age, but they are moderate and minimally impact function of organs in the elderly patients [9]. As pointed out by Grassi et al. [20], no digestive diseases are seen only in geriatric patients, but compared with younger persons the prevalence of functional disorders affecting gastrointestinal system is significantly higher in the elderly. Changes in the aging gastrointestinal tract include decreased saliva production, generalized slowing of peristalsis, reduced pepsin, bicarbonate and pancreatic enzymes (lipase, chymotrypsin, amylase) secretions, decreased biodiversity and endangered stability of the intestinal microbiota $[8,9,21]$. Some of these changes, like reduced gastric motility and a decline in bicarbonate and prostaglandin production, may raise the risk of adverse drug events. Slowed peristalsis in the small and large intestine put older people at a higher risk for constipation [9]. On the other hand, many of the elderly people have special risk factors for developing travellers' diarrhoea, e.g. diabetes and taking histamine-blockers or proton pump inhibitors, resulting in a reduction of the host defence of gastric acidity [13, 22]. There is also a higher probability of developing complications of travellers' diarrhoea in this group of patients [22].

\section{IMMUNE SYSTEM}

One of the most noticeable changes associated with the immune system ageing is thymic involution, which results in a decreased output of mature T cells. B cells immune response is also impaired due to age related changes $[5,10]$. As a result, the immunity conferred through vaccinations received in the past may become impaired, a response to more recently received vaccines may be decreased and the duration of immunity is generally shorter. Also noteworthy are the changes in cytokine production, including lower level of Th1 cytokines (IL-12 and IFN-G) and an increased production of Th2 cytokines (IL-10 and TGF-B) [23]. Due to the dysfunction of the immune system elderly patients are more susceptible to infections, e.g. cosmopolitan diseases like pneumonia as well as travel-associated illnesses, e.g. malaria $[5,24]$.

\section{SKIN}

Changes in the skin are the most visible signs of ageing. They are especially pronounced in the uncovered parts of the body: the face, neck, upper part of the chest, hands and forearms, and result in the alteration of people's physical appearance $[8,9]$. Apart from the aesthetic aspect, skin ageing may have a negative effect on skin function and therefore it is more than barely a cosmetic problem $[9,25]$. The degenerative changes that occur with age include a decrease in the amount of collagen, elastin, connective tissue and fat $[9,25,26]$. Clinical presentations of age-related skin degeneration may indeed be very serious and may even manifest as basal and squamous cell carcinoma and malignant melanoma [25]. Due to a decrease in melanocytes, skin is easily damaged by exposure to the sun, and so elderly travellers become increasingly vulnerable to the UV light $[9,26]$. Therefore, it is crucial for the elderly to remember about adequate skin protection, especially while travelling to sunny destinations.

\section{MUSCULOSKELETAL SYSTEM}

Ageing is associated with natural changes in body composition. Fat mass increases while muscle mass and total body water decreases. These changes are responsible for reduce muscle strength and growing muscle fatigue in elderly people $[9,27]$. Another common problem of geriatric patients is osteoporosis, which may result in bone fractures [8]. All of that put elderly people at higher risk for falls, especially as travellers visit unfamiliar destinations $[9,10]$.

\section{POLYPHARMACY}

Another problem of the elderly is polypharmacy due to multimorbidity, as one or more medications can be used to treat single disease [28]. Although polypharmacy is not consistently defined in the literature, it is usually explained as taking 5-6 medications daily [29]. As Masnoon et al. [28] pointed out, numerical only definition is the most popular, but even the numerical definitions vary to a large extent; some define polypharmacy as the use of two or more drugs concurrently while others as the use of above 11 medications at the same time. There are also other ways to explain polypharmacy, e.g. definitions which take into account both the number of medicines taken and also the length of therapy or treatment setting, as well as descriptive definitions. 
Differences in the understanding of the term 'polypharmacy' may be the reason why its prevalence varied a great deal between different studies. Kim et al. [29], in South Korea, reported that polypharmacy was found in $86.4 \%$ of elderly patients, whereas in the Finnish study performed by Jyrkkä et al. [30], polypharmacy was only seen in $33 \%$ of patients. It is important to note that the use of medications can impact morbidity and quality of life and taking multiple of drugs may have many negative consequences [31, 32]. Polypharmacy has been associated with increased risk of adverse drug events, medication non-adherence or drug-interactions [32]. According to Doan et al. [33], the risk of drug-drug interactions increase with the number of medicaments taken: when a patient is taking 5-9 drugs daily, it is estimated at $50 \%$, and in patients taking 20 or more medications, the risk increases to $100 \%$. It is essential to remember about potential drug interactions that may occur during travel [22]. Drug-drug interactions between antibiotics used for empiric therapy of travellers' diarrhoea, antimalarials used for chemoprophylaxis, altitude illness prevention drugs and other common medications prescribed to the elderly should be considered prior to travel $[10,22]$. Another important issue is that many elderly travellers fail to take prescribed drugs, especially diuretics during travel [26], and leave their emergency or routine medications at home [19].

\section{MOST COMMON TRAVEL-ASSOCIATED DISEASES}

\section{ARTHROPOD-BORNE DISEASES}

Malaria is one of the most widespread infectious diseases in the world. It is a mosquito-borne disease caused by five species of protozoa that belong to the Plasmodium genus: $P$. falciparum, $P$. vivax, P. ovale, P. malariae and $P$. knowlesi. According to the World Health Organisation (WHO), there were 216 million registered malaria cases and 445 thousand deaths in 2016 [34]. In an observational study that analyse the trends in the prevalence of imported malaria in London [35], of a total of 15,473 cases reported between 2000 and 2004, 5.9\% were seen in patients older than 60 years; between 2005 and 2009 the prevalence of malaria in this group reached $7.7 \%$ and between 2010 and 2014 $-8.7 \%$. In the study performed by Allen et al. [36], 5.9\% of the cases reported between 2002 and 2012 were found in patients aged 65 years or older. Travellers coming from non-endemic countries visiting endemic regions are at a particularly high risk for malaria acquisition, the mortality rates associated with malaria are also higher in this group of travellers [37]. What is more, because of physiological changes and multimorbidity elderly travellers are at an increased risk of serious complications of malaria and other travel-related diseases [36]. A questionnaire-based survey carried out at
Amsterdam Airport Schiphol showed that travellers aged 60 and over are at a similar risk of malaria as younger persons [38]; however according to Checkley et al. [39], patients over the age of 65 are approximately 10 times more likely to die from malaria in comparison with younger patients. Also, Allen et al. [36] found that the length of hospital stay (LOS) increases with age - the median LOS was 8 days for the elderly compared to a median LOS of 3 days in younger groups. While the median time from consultation to diagnosis was 2 days and ranged between 0 and 35 days resulting in a delay of treatment initiation. It is essential to note that age, delayed diagnosis and a delay in treatment initiation are significant risk factors for fatal malaria. Therefore it is important to take patient's history very carefully, especially if a returned traveller presents with fever - this can facilitate early diagnosis, improve clinical outcomes and decrease morbidity and mortality. However, due to multiple comorbidities presenting features vary with age, so the identification of imported infection may be challenging [36].

Dengue is a disease caused by any one of the four related viruses and is transmitted by mosquitoes. According to the WHO [40], 390 million dengue infections occur every year. Dengue virus causes a wide variety of diseases from a self-limiting febrile illness - dengue fever to a severe, life-threatening diseases known as dengue haemorrhagic fever (DHF) or dengue shock syndrome (DSS). DHF and DSS usually affect children living in endemic areas and infrequently adults, especially travellers [41]. In the study performed by Rowe et al. [42], 4.4\% of the 6989 cases were found in patients aged 60 or older, although in other studies the prevalence of dengue in patients older than 65 years ranged between $4.2 \%$ and $8.7 \%[43,44]$. Although the number of cases in the elderly is relatively low, a Taiwanese study showed that $69.9 \%$ of all patients hospitalised for dengue and $85.3 \%$ of dengue patients admitted to intensive care units were over 60 [45]. Compared with younger patients, the classical symptoms including a headache, rash, pain are less common in the elderly. Despite this fact, the time from consultation to dengue diagnosis was similar in the two groups. It is important to mention that that elderly stayed in hospital longer (5 vs. 4 days) [42]. Another example of an arthropod-borne illness is Chagas disease (CD). It is most commonly transmitted to humans by triatomine bugs and is caused by the Trypanosoma cruzi parasite. It is estimated that eight million people are infected, particularly in Latin America; however, more than 300,000 people with $T$. cruzi infection live in the United States [46]. In the study performed by Pereira et al. [47], of the total number of 411 patients $23.6 \%$ were older than 60 years and between 2005 and 2013 the prevalence of $C D$ in the elderly was steadily increasing. In this group of 
patients the cardiac form of CD was predominant, followed by the mixed and digestive forms $[47,48]$. The elderly affected by $C D$ show a greater number of ageing-associated diseases. What is more, the clinical interaction between $C D$ and other age-related illnesses may increase mortality and morbidity and may reduce the quality of life. Indeed, morbidity rates among the elderly are higher in comparison with other age groups [47-49]. Pereira et al. [49] highlighted that the elderly patients with CD need special attention from healthcare providers and that further studies in this group of patients are necessary. Leishmaniasis is a disease caused by an infection with Leishmania parasites and is spread by the bite of phlebotomine sand flies. There are three clinical forms of leishmaniasis: visceral (VL), cutaneous and mucocutaneous. Leishmaniasis is found in the tropics, subtropics and southern Europe. However, $90 \%$ of VL cases (the most severe form of leishmaniasis) are reported from several countries only; these countries however are popular travel destinations, e.g. Brazil, India or Nepal [23, 50, 51]. According to Montalvo et al. [52] high human global mobility is one of the factors which have led to an increase in the number of cases in non-endemic regions such as Cuba. Also noteworthy is that lethality rates for VL have risen in many areas over the past 10 years, especially in patients over 50 years. A higher lethality is connected with late diagnosis, atypical presentations and multimorbidity. Despite the fact that multidrug therapy is associated with higher risk of drug interactions and cumulative toxicity, it was not found to influence the mortality rate in VL patients [23].

\section{TRAVELLERS' DIARRHOEA}

Travellers' diarrhoea (TD) is the most common travel-related health problem which affects $20-50 \%$ of international travellers [53]. It is defined as three or more loose stools occurring within 24 hours associated with one or more enteric symptom. TD is most common among people travelling to South-East Asia, Sub-Saharan Africa and Latin America. Bacterial infections cause $50-80 \%$ of TD cases. Traditionally, enterotoxigenic Escherichia coli is considered to be the major causative pathogen of TD. Other etiological factors include enteroaggregative E.coli, enteropathogenic E.coli, Shigella, Salmonella, and especially in South-East Asia - Campylobacter. Less common are viral and protozoal pathogens $[53,54]$. TD is usually a mild, self-limiting disease, and it generally resolves spontaneously after 3-4 days. However, it may be the cause of activity limitation leading to loss of business or vacation days. In some cases the clinical picture of TD can be more severe - about $10 \%$ of travellers affected by TD seek medical care and hospitalisation is required in up to $3 \%$ of cases [54]. Acute TD was shown to be less frequent in the elderly compared to younger travellers [11]; however, it is important to highlight that some group of elderly patients are at a higher risk of TD sequelae. For example, travellers with age-associated diseases, e.g. heart failure or renal insufficiency are more vulnerable to dehydration and electrolyte imbalance, which can lead to the exacerbation of the underlying disease. TD can also lead to persistent gastrointestinal symptoms like post-infectious irritable bowel syndrome (PI-IBS) or other post-infectious sequelae like Guillain-Barre syndrome or reactive arthritis. Generally, older travellers are at lower risk of PI-IBS in comparison to the younger ones; nevertheless, patients with severe disease are at an increased risk of PI-IBS. What is more, medicines commonly used for travellers' diarrhoea, such as antibiotics and anti-motility agents, may impact TD's potential to develop into PI-IBS [10, 54]. Guillain-Barre syndrome, typically associated with a Campylobacter infection, most commonly affects young adults and the elderly and may lead to permanent disability or even death [54].

\section{HIGH-ALTITUDE ILLNESS}

In 2013, more than 100 million people travelled to areas where altitude illness is likely to occur. It is important to note that high-altitude cities, like Cusco, La Paz or Lhasa, are becoming popular tourist destinations also among elderly travellers and travellers with chronic illnesses [55]. There are three main forms of the disease: acute mountain sickness (AMS), high-altitude pulmonary oedema (HAPE) and high-altitude cerebral oedema (HACE). AMS is not life-threatening, although it may lead to more severe conditions: HAPE and HACE. Risk factors for developing high-altitude illness include quick ascent, previous history of AMS, HAPE or HACE, inadequate fluid intake and climbing up despite signs which may indicate poor tolerance of height $[55,56]$. According to Gautret et al. [11], elderly people may be at higher risk of developing high-altitude illness. On the other hand, other studies showed that older travellers with no co-morbidities have no increased risk of the disease [55], although the risk of HAPE may be elevated in individuals over the age of 65 [56]. Contraindications to travel to areas located at high altitude include common age-related diseases such as severe obstructive pulmonary disease, symptomatic pulmonary hypertension, severe or uncontrolled heart failure or severe ischaemic heart disease $[55,56]$. The best way to avoid AMS is primary prevention, which include appropriate physical preparation, slow gradual ascent, pre-acclimatisation, adequate fluid intake and avoiding overexertion. In the case of patients with a history of altitude illness or travellers who are not able to follow the recommendations above medications may be a useful adjunct to the prevention of high-altitude illness [55-57]. 


\section{PREVENTION OF ELDERLY TRAVELLERS}

\section{PRE-TRAVEL CONSULTATION}

Pre-travel counselling has become an essential part of comprehensive family medicine over the last years [58]. The aim of the pre-travel health consultation is to prepare travellers for a safe and healthy trip. A healthcare provider familiar with travel medicine should assess the risk of travel and provide proper guidance. These consultations give the opportunity not only to provide a patient with specific information but also to administer all necessary vaccinations and prescribe medications for self-treatment of diarrhoea or for malaria chemoprophylaxis. The effectiveness of a pre-travel consultation depends both on expert advice and patient compliance [10, 58-60]. Elderly people, especially with comorbidities including cardiovascular disease or diabetes mellitus are at a higher risk of travel-associated diseases, therefore they require greater attention [59]. In the study performed by Gautret et al. [11], the proportion of patients who sought pre-travel advice was $42.2 \%$ among patients aged 60 and over, and $51.7 \%$ in travellers aged 18-45 years. According to Jaeger et al. [5], almost $10 \%$ of patients who obtained pre-travel health advice were people aged 60 or above. This group of patients consulted a travel clinic earlier compared to middle-aged adults. The most common reasons for not seeking pre-travel advice included: not being aware of such a necessity, the belief that prevention measures undertaken by a patient would be sufficient, financial limitations and language barriers [61].

\section{NON TRAVEL-RELATED VACCINES}

As mentioned before, a pre-travel consultation gives an opportunity to administer routine vaccines. Immunisations are extremely important for older adults, due to their increased vulnerability to infections. Although, it must be remembered that immunogenicity and efficiency of most vaccines are decreased due to age-associated changes in the immune system and there are no alternative schedules to compensate for the immunosenescence for the majority of vaccines [10,62]. Routine influenza vaccination is particularly recommended in the elderly as they are highly vulnerable to influenza and are at a higher risk of developing serious complications from the disease. Approximately $90 \%$ of influenza-associated deaths occur among the elderly [63]. Influenza vaccination should be considered prior to travel to tropical and subtropical countries. According to Mutsch et al. [64], influenza is the most common vaccine-preventable infection in travellers to tropical or subtropical areas and immunisation should be recommended to the traditional risk groups, e.g. senior travellers and patients with multiple comorbidities. Several studies demonstrated clinical efficacy of influenza immunisation in the elderly, resulting in a re- duced number of influenza-associated hospitalisations and deaths, although generally, seroprotection rates are lower in people age 65 and older $[10,62]$. In some cases it may be reasonable to prescribe elderly travellers a self-treatment course of zanamivir or oseltamivir, in case the vaccine is ineffective [10]. Community-acquired pneumonia is another major health issue affecting the elderly globally; Streptococcus pneumoniae being the leading cause of CAD. Most industrialised countries recommend routine vaccination to all adults age 65 and older. Both the 23-valent pneumococcal polysaccharide vaccine (PPSV23) and the 13-valent pneumococcal conjugate vaccine (PCV13) are approved for the use $[65,66]$. Systematic review and meta-analysis performed by Falkenhorst et al. [66] showed that PPSV23 is indeed effective, although previous studies had demonstrated its ineffectiveness. What is more, PPSV23 should be recommended for routine vaccination, due to its broader serotype coverage compared to PCV13. As pointed out by Lee et al. [10], there is not enough information regarding the prevalence of pneumococcal pneumonia in travellers; however, a pre-travel counselling visit is a good opportunity to ensure that elderly travellers are aware of the current vaccination recommendations. It is estimated that more than $90 \%$ of adults are latently infected with varicella zoster virus (VZV) and are at risk for herpes zoster. The primary infection is especially likely to occur in children; however, due to immunosuppression or immunosenescence viral reactivation may occur at any age and may develop into herpes zoster. The disease is most frequent in adults aged 50 and over and nearly $50 \%$ of all cases affect individuals older than 85 years of age. Elderly people are also at a higher risk of developing complications from herpes zoster, such as postherpetic neuralgia. An attenuated live-vaccine has been licensed for use in adults above 50 years old and is recommended to all adults older than 60 in some countries. The vaccine reduced the incidence of herpes zoster by $51.3 \%$ and showed $66.5 \%$ efficacy against postherpetic neuralgia in patients; however, its overall efficacy decreased with age. It is important to remember that immunisation with live vaccines is contraindicated in immunosuppressed patients [62, 65, 67]. In the study performed by Lal et al. [67], the overall efficacy of a new adjuvanted herpes zoster subunit vaccine was $97.2 \%$. Little is known about the herpes zoster prevalence among travellers; however, due to its high prevalence among the elderly it is possible that an outbreak may occur while travelling. It is reasonable to administer a full course of the vaccine to senior travellers, and if the last dose had been administered more than 8 years prior to travel, a booster dose is recommended [10]. Vaccination against tetanus, pertussis and diphtheria is advised to be given to all adults. In many countries patients are recommended to receive a booster dose every 10 years; in some 
countries booster intervals are shortened for patients over the age of 65 as the response to the vaccination becomes impaired with age $[62,65]$. As Lee et al. [10] pointed out, there is little information about the incidence of tetanus, diphtheria and pertussis in travellers. Due to the measles, mumps and rubella (MMR) outbreaks in the United States there has recently been a focus on immunization against MMR; , however, the MMR vaccine is not essential for the elderly travellers.

\section{TRAVEL-RELATED VACCINES}

A single dose of live-attenuated yellow fever vaccine provides lifelong immunity in the majority of patients. In the study performed by Jaeger et al. [5], it was the most often administered vaccine in the group of elderly travellers. The vaccine is highly effective; however, in some groups of patients, e.g. individuals over 60 years old, the risk of developing a reaction to the immunisation may be elevated [68]. Side effects are rare, but can be severe, including yellow fever vaccine-associated viscerotropic disease (YEL-AVD) and yellow fever vaccine-associated neurologic disease (YEL-AND) which may manifest as meningoencephalitis, disseminated encephalomyelitis or Guillain-Barre syndrome [10]. The risk of YEL-AVD or YEL-AND is approximately 0.5/100,000 administered doses. However, the risk of YEL-AVD in patients aged over 60 years is about 1.0-1.1/100,000 distributed doses, rising to 2.3-3.2/100,000 in individuals aged above 70 [5]. Another study also showed that the risk of serious systemic adverse events was significantly higher in individuals aged 65 years or older in comparison to the 25-44 age group. Additionally, a majority of deaths from YEL-AVD were seen in adults aged 45 years and above [69]. Indeed, more travellers were found to have died from YEL-AVD than yellow fever itself [10]. It is the role of a healthcare provider, in collaboration with a patient, to decide whether the benefits from vaccination outweigh the risks $[10,68]$. Hepatitis $A$ is the second most common vaccine-preventable disease among non-immune travellers [10] and international travel was identified to be the major risk factor for hepatitis $A$ virus (HAV) acquisition [70]. The incidence rate varies between 6.0 and 30.0 cases per 100,000 months of stay in virtually all developing countries [71]. In children a majority of infections are asymptomatic; however, in adults the infection often leads to acute hepatitis that may result in prolonged incapacitation or to acute liver failure [72]. In the study performed by Mutsch et al. [71], 5.3\% of all hepatitis A cases occurred in the group of patients older than 60 years. There are several effective hepatitis $A$ vaccines with a very low risk of side effects [70]. However, due to their immunosenescence, individuals over 50 years are recommended to receive two doses of the vaccine. The seroprotective rate was found to be $65 \%$ after the first dose and $97 \%$ after receiving the booster (in the group of patients aged 18 to 45 years it was $100 \%$ after a single dose) [10]. Hepatitis A vaccine is recommended to high risk groups, e.g. all people who travel to endemic areas or those who have chronic liver disease [70]. In the study performed by Jaeger et al. [5], travellers over the age of 60 received the vaccine significantly less often compared to younger groups. Hepatitis B is one of the most common vaccine-preventable travel-associated infectious diseases. It is estimated that approximately 240 million people worldwide are infected with hepatitis $B$ virus (HBV) and approximately 1 million people die each year from HBV-related illness [73]. The risk of acquiring HBV during the travel is relatively low (about 0.2 to 0.6 per 1000 person-months). However, elderly people are more likely to acquire travel-associated HBV infection as they require medical treatment more often than younger travellers and because medical tourism is growing in popularity [74]. About $20 \%$ of patients with hepatitis B infection need to be hospitalised and the risk is doubled in individuals 50 years and older. What is more, elderly patients with co-morbidities, such as liver or renal disease or diabetes are at a higher risk of developing complications from hepatitis B [75]. However, the risk of chronic HBV infection in people over 40 years of age is lower in comparison to young adults (6\% vs. $8 \%$ ) [76]. The hepatitis B vaccine is recommended to all unvaccinated travellers going to regions with intermediate to high prevalence of chronic hepatitis B; however, only 15-30\% of international travellers had received a full vaccination course prior to travel [73]. The Swiss study showed that hepatitis $B$ vaccination was administered significantly less often to senior travellers [5]. It is important to note that age is the strongest predictor of vaccine response. In the study performed by Van Der Meeren et al. [75], vaccine protection rate was above $90 \%$ in individuals aged $20-49$ years and only $64.8 \%$ in those aged 65 years or more. Another study showed that only $75 \%$ of the people vaccinated after the age of 60 years develop protective antibody levels [77]. For this reason, further studies are necessary to establish if elderly patients would benefit from additional or higher doses of the hepatitis $B$ vaccine [75]. Typhoid is one of the most commonly diagnosed vaccine-preventable illness in returned international travellers. The incidence rate of typhoid infection is estimated at $1-10$ cases per 100,000 travellers to developing countries [78]. The effectiveness of typhoid vaccine has been reported to be $65 \%$ to $80 \%$ $[79,80]$ and no significant difference in efficiency by age group has been noticed [79]. According to Lee et al. [10], typhoid vaccine should be considered in elderly travellers to endemic regions as an effective way of preventing the disease. Rabies is a preventable fatal disease that causes 50,000-55,000 deaths worldwide each year. However, in the decade prior to the study performed by Gautret et al. [81] 
only 22 cases have been confirmed in travellers. Many studies confirmed safety and immunogenicity of the rabies vaccine with the overall seroconversion rate being $94.5 \%$ in patients up to age $65[81,82]$. WHO recommends the rabies vaccine to all travellers [10]. Other travel-related vaccines include meningococcal, polio or Japanese encephalitis vaccines. The conjugated meningococcal vaccine needs to be considered in all senior travellers. Moreover, in some settings, e.g. the Hajj pilgrimage, the vaccine is compulsory for all pilgrims. The polio vaccine should also be considered in this group of patients and may be a requirement for travellers going to endemic areas. The Japanese encephalitis vaccine should be given to senior patients that travel to endemic regions [10].

\section{MALARIA PREVENTION}

Travellers to malaria-endemic regions should be informed about the effective methods of protection against insect bites (e.g. covering arms and legs, using insect repellents, e.g. DEET, and permethrin impregnation) [83]. Returned travellers who develop a febrile illness are recommended to seek prompt medical assistance. The recommendations concerning malaria prophylaxis refer to all travellers, regardless of age [10]. Yet, a study carried out by Alon et al. [13] revealed that travellers over the age of 60 used insect repellents significantly less often than travellers aged $20-30$ years ( $46.6 \%$ vs. $60 \%$ ). Chemoprophylaxis is likewise essential to prevent malaria and when taken properly, it is highly effective. Main options for malaria chemoprophylaxis include atovaquone/proguanil, doxycycline, mefloquine and chloroquine. It should be offered to all patients travelling to endemic areas, when an exposure to malaria is time-limited and acceptable compliance is realistic. All of these drugs are well tolerated by older patients; however, it is important to remember about possible side effects, contraindications and drug-drug interactions [10,83]. Atovaquone/proguanil is the most frequently prescribed chemoprophylactic agent in the group of elderly travellers [13]. It has very few drug-drug interactions which is particularly important considering the fact that polypharmacy is increasingly common in elderly patients. However, travel medicine providers should be aware of potential interactions between atovaquone/ proguanil and metoclopramide or warfarin. Possible side effects may include headache and abdominal pain, yet, these are very rare. There are not pharmacokinetics differences between elderly and younger patients and dosing regimens do not need to be modified, regardless of age. However, the drug cannot be used in patients with severe renal insufficiency (creatinine clearance $<30 \mathrm{~mL} / \mathrm{min}$ ) [10, 84]. Doxycycline may be used in the elderly for the prevention of malaria; however, it is associated with many inconvenient side effects, including oesophageal irritation, ulceration or photodermatitis. What is more, dietary and supplement restrictions may be necessary while taking doxycycline. It is also important to remember about drug-drug interactions between doxycycline and calcium salts, carbamazepine, phenytoin or warfarin. Another drug commonly used for the prevention of malaria is chloroquine, although due to high Plasmodium resistance to the drug, it has been used in limited settings. Moreover, long-term use of chloroquine is associated with retinopathy and macular degeneration. Interactions with amiodarone, sotalol, propranolol, metoprolol, carvedilol, fluoxetine, paroxetine, ciprofloxacin, levofloxacin, azithromycin or digoxin are likely to occur, and therefore, the drug is not recommended to the elderly [10, 83]. Mefloquine is more often recommended to younger travellers [13], especially those who travel to endemic areas for a longer period of time [83]. According to Lee et al. [10], it should not be used in elderly patients with cardiac conduction disease or neuropsychiatric disorders. However, as Hahn et al. [83] pointed out, the risk of neuropsychiatric side effects is in general overstated. Nevertheless, there are many drug-drug interactions between mefloquine and carbamazepine, phenytoin, citalopram, fluoxetine, azithromycin, verapamil, carvedilol or warfarin [10]. Main reasons for declining to take chemoprophylaxis include cost, lack of awareness and scepticism about therapy efficiency, inappropriate assessment of personal risk or failure to access drugs before travel [35]. It is essential to highlight that patients whose main reason to travel is visiting friends and relatives are less likely to take antimalarial prophylaxis [85]. Chemoprophylaxis is effective only with appropriate patient compliance [83]. In the study performed by Hill [86], 80\% of travellers stated that they were fully compliant with their chemoprophylaxis regimen. Non-compliance affects all age groups; however, elderly patients generally comply better with the prescribed drug regimen According to Alon et al. [13], $60.7 \%$ of individuals at the age of 60 and above were completely compliant compared to $33.8 \%$ travellers aged 20-30 years. The main reason for not complying with recommended regimen in the group of elderly travellers was the statement that "nobody takes these drugs anyway" (19.6\%). For $7.1 \%$ of the elderly the main reasons included possible side effects, $6.2 \%$ of senior travellers stated that did not believe in effectiveness of the drugs and finally, for $2.6 \%$ of them the regimen was too inconvenient.

\section{TRAVELLERS' DIARRHOEA PREVENTION AND EARLY SELF-TREATMENT}

Options for preventing travellers' diarrhoea, in travellers of all ages, include avoidance of unsafe food and drinks, frequent hand washing, vaccination, non-antibiotic and antibiotic interventions. As mentioned before, senior travellers tend to comply with travel health precautions better than 
the younger ones $[13,53,54]$. The orally administered, killed whole-cell cholera vaccine has more than $90 \%$ efficiency against Vibrio cholerae and due to the antigenic similarity it may also reduce enterotoxigenic Escherichia coli TD. Effectiveness of probiotics in preventing TD is limited. Bismuth subsalicylate can provide up to $65 \%$ protection against TD; however, it is important to remember about potential drug-drug interactions, especially in elderly travellers. It may also reduce the absorption of doxycycline, used for malaria chemoprophylaxis. Frequent administration of bismuth subsalicylate may lead to decrease in the overall compliance [54]. Antibiotics are up to $90 \%$ effective in preventing TD. Antibiotic options for prevention of TD include fluoroquinolones (levofloxacin and ciprofloxacin), rifaximin and azithromycin. Despite high efficiency, antibiotic prophylaxis has a number of disadvantages which limit its usefulness, especially among elderly travellers. The disadvantages include possible side effects, cost, drug interactions, the risk of acquiring Clostridium difficile infection or antibiotic resistant microorganisms [53, 54, 87]. Rifaximin use is usually limited by cost. Azithromycin and levofloxacin (but not ciprofloxacin) increase the risk of ventricular arrhythmia and cardiovascular death and may lead to electrolyte imbalance in cases of severe diarrhoea. In light of the data, the above-mentioned antibiotics should be avoided in patients with QTc prolongation and are not recommended to patients with underlying heart disease [54]. What is more, azithromycin and fluoroquinolones may interact with many other drugs, e.g. citalopram, fluoxetine, escitalopram, amiodarone, dronaderone, atorvastatin, simvastatin, warfarin and multivitamins or minerals [10]. In general, antibiotic dosage regimens need to be suitably modified for use in the elderly [87]. Initial symptoms of TD should be an indication to self-treatment based on oral rehydration therapy, this is particularly important for the elderly. Although in general increasing the amount of fluid with clean water is enough, WHO-formulated oral rehydration salts are recommended, especially for moderate and severe dehydration and in vulnerable groups of patients, i.e., older travellers. In mild cases of nondysenteric TD, antimotility agents such as loperamide can be used. If TD is moderate or severe, symptoms worsen or do not subside after 24 hours of loperamide usage, a combined therapy with loperamide and antibiotics is needed. Antibiotics for early self-treatment are generally the same as these used for TD prevention [53,54]. Patients should seek medical assistance if symptoms persist or worsen despite self-treatment, if TD is accompanied by persistent fever, chills, bloody diarrhoea, abdominal pain, intractable vomiting, or if accurate oral rehydration therapy is impossible. Senior patients with underlying diseases who develop TD are recommended to look for medical care earlier than other patients, due to increased risk of severe and complicated TD [54]. Antibiotic prophylaxis should be reserved for high risk travellers, e.g. immunocompromised patients [54]. While antibiotic treatment of TD in elderly travellers should only be limited to severe cases. There are several reasons for these recommendations. Firstly, senior travellers are at higher risk of extended spectrum beta-lactamase (ESBL) carriage and colonisation with other multi-drug resistant bacteria. ESBL carriage may, in turn, lead to complications such as the urinary tract infections or prostatitis [10]. What is more, although antibiotics and anti-motility agents are effective in the treatment of TD, their application may increase the risk of developing PI-IBS [54]. Antibiotic use during travel has also been associated with increased risk for $C$. difficile infection [88]. In light of these findings, antibiotic options may only be used for the treatment of TD when it is absolutely necessary.

\section{ALTITUDE SICKNESS PREVENTION}

The most commonly prescribed drug to prevent AMS is acetazolamide. It decreases the metabolic alkalosis, acts as a mild diuretic thus reducing oedema and it is the only one drug available which facilitates acclimatisation. Acetazolamide should be used the night before planned ascent until the beginning of descent or until a person has been at the target elevation for 2-3 days. The effectiveness of acetazolamide $250 \mathrm{mg}$ daily in decreasing AMS has been demonstrated; however, it is important to remember about contraindications and drug interactions, especially in the group of elderly travellers $[55,89]$. The study performed by Alon et al. [13] demonstrated that elderly travellers used acetazolamide for high-altitude illness prevention significantly more often than younger travellers (58\% vs. $8.3 \%$ ). Allergies, previous anaphylactic reactions, significant hepatic and renal dysfunction, severe chronic lung dysfunction are contraindications to acetazolamide. As mentioned before, common problem of elderly patients is polypharmacy and as a consequence drug-drug interactions. For example, patients on high-dose acetylsalicylic acid (325 mg daily) are at higher risk of metabolic acidosis while using acetazolamide. There is also elevated risk for metabolic acidosis and nephrolithiasis during concomitant use of acetazolamide and brinzolamide, dorzolamide, topiramate or zonisamide. Travel medicine providers should be also aware of a possible interaction between acetazolamide and loop diuretics, tramadol, oxycodone, codeine or metformin. Side effects of acetazolamide include paraesthesia, polyuria, rash and dysgeusia $[10,55,89]$. In case of contraindications or intolerance to acetazolamide, dexamethasone is a well-studied alternative. Contrary to acetazolamide it only conceals the symptoms of AMS by reducing the inflammation. Dexamethasone should not be used for more than 7 consecutive 
days due to as the drug may produce Cushingoid effects or adrenal suppression. Furthermore, it may have significant side effects, e.g. insomnia, unmasking of diabetes, peptic ulceration and gastrointestinal bleeding. In recent years, the effectiveness of inhaled budesonide has been confirmed for the prevention of AMS. The drug exhibits fewer side effects compared to dexamethasone [55, 89]. Nifedipine is the firstline drug for prevention and treatment of HAPE. Side effects of this medication include AMS-like symptoms: headache, swollen ankles and constipation. Particularly noteworthy are significant interactions with drugs metabolised by the cytochrome CYP3A4 [55]. Recent studies have suggested that sumatriptan, gabapentin and ibuprofen may be effective at preventing high-altitude illness; however, further studies should be carried out $[55,89]$.

\section{CONCLUSIONS}

Prolonged life expectancy and improved quality of life are associated with an increase in the number of elderly travellers. Although older patients represent a relatively small proportion of international travellers, they demand greater attention because of the multimorbidity and polypharmacy which are seen in many older people. Ageing has a variable effect on common travel-related illnesses. Pre-travel counselling performed by a provider familiar with travel medicine is desirable in the group of elderly travellers and is a good opportunity to administer both non travel-related and travel-associated vaccines, bearing in mind decreased immune response in the elderly. Advanced age also affect the management of malaria prevention, travellers' diarrhoea and altitude illness. In light of these findings, every case should be considered on an individual basis.

\section{REFERENCES}

1. World Tourism Organization. UNWTO Tourism Highlights: 2018 Edition. https://www.e-unwto.org/doi/pdf/10.18111/97892844 19876 (Accessed: 30 Oct 2018).

2. The World Bank. Population ages 65 and above (\% of total). https://data. worldbank.org/indicator/SP.POP.65UP.TO.ZS (Accessed: 28 Oct 2018).

3. Kontis $\mathrm{V}$, Bennett JE, Mathers $\mathrm{CD}$, et al. Future life expectancy in 35 industrialised countries: projections with a Bayesian model ensemble. Lancet. 2017; 389(10076): 1323-1335, doi: 10.1016/ S0140-6736(16)32381-9, indexed in Pubmed: 28236464.

4. Eurostat. Mortality and life expectancy statistics. https://ec.europa. eu/ eurostat/statistics/explained/index.php/Mortality_and_life_ expectancy_statistics\#Life_expectancy_at_birth_increased_ in_2016 (Accessed: 30 Oct 2018).

5. Jaeger VK, Tschudi N, Rüegg R, et al. The elderly, the young and the pregnant traveler: A retrospective data analysis from a large Swiss Travel Center with a special focus on malaria prophylaxis and yellow fever vaccination. Travel Med Infect Dis. 2015; 13(6): 475-484, doi:10.1016/j.tmaid.2015.10.001, indexed in Pubmed: 26526774.

6. Utama R, Bagus P. Motivation and Satisfaction of Senior Tourists for Traveling Overseas. 13th International Conference on
Academy and Business, Bali, Indonesia. 2012; September, doi: 10.13140/2.1.1475.2008.

7. Nimrod G. Retirement and tourism Themes in retirees' narratives. Ann Tourism Res. 2008; 35(4): 859-878, doi: 10.1016/j.annals.2008.06.001.

8. Boss GR, Seegmiller JE. Age-related physiological changes and their clinical significance. West J Med. 1981; 135(6): 434-440, indexed in Pubmed:7336713.

9. King M, Lipsky MS. Clinical implications of aging. Dis Mon. 2015; 61(11): 467-474, doi: 10.1016/j.disamonth.2015.09.006, indexed in Pubmed:26497929.

10. Lee TK, Hutter JN, Masel J, et al. Guidelines for the prevention of travel-associated illness in older adults. Trop Dis Travel Med Vaccines. 2017; 3: 10, doi: 10.1186/s40794-017-0054-0, indexed in Pubmed: 28883980.

11. Gautret P, Gaudart J, Leder K, et al. GeoSentinel Surveillance Network. Travel-associated illness in older adults (>60 y). J Travel Med. 2012; 19(3): 169-177, doi: 10.1111/j.1708-8305.2012.00613.x, indexed in Pubmed: 22530824.

12. Eurostat. People in the EU - statistics on an ageing society. https:// ec. europa.eu/eurostat/statistics-explained/index.php/People_in_ the_EU_-_statistics_on_an_ageing_society (Accessed: 10 Nov 2018).

13. Alon D, Shitrit P, Chowers M. Risk behaviors and spectrum of diseases among elderly travelers: a comparison of younger and older adults. J Travel Med. 2010; 17(4): 250-255, doi: 10.1111/j.17088305.2010.00425.x, indexed in Pubmed: 20636598.

14. Eurostat. Tourism trends and ageing. https://ec.europa.eu/eurostat/ statistics-explained/index.php/Tourism_trends_and_ageing (Accessed: 31 Oct 2018).

15. Sundling C, Berglund B, Nilsson ME, et al. Overall accessibility to traveling by rail for the elderly with and without functional limitations: the whole-trip perspective. Int J Environ Res Public Health. 2014; 11(12): 12938-12968, doi: 10.3390/ijerph111212938, indexed in Pubmed: 25514149.

16. Paneni F, Diaz Cañestro C, Libby P, et al. The Aging Cardiovascular System: Understanding It at the Cellular and Clinical Levels. J Am Coll Cardiol. 2017; 69(15): 1952-1967, doi: 10.1016/j. jacc.2017.01.064, indexed in Pubmed: 28408026.

17. Gander J, Sui X, Hazlett L, et al. Factors related to coronary heart disease risk among men: validation of the Framingham Risk Score. Prev Chronic Dis. 2014; 11: E140, doi: 10.5888/pcd11.140045, indexed in Pubmed: 25121352.

18. Joy M. Cardiovascular disease and airline travel. Heart. 2007; 93(12): 1507-1509, doi: 10.1136/hrt.2007.134247, indexed in Pubmed: 18003680.

19. McIntosh IB. Health hazards and the elderly traveler. J Travel Med. 1998; 5(1): 27-29, indexed in Pubmed: 9917179.

20. Grassi M, Petraccia L, Mennuni G, et al. Changes, functional disorders, and diseases in the gastrointestinal tract of elderly. Nutr Hosp. 2011; 26(4): 659-668, doi: 10.1590/S021216112011000400001, indexed in Pubmed: 22470008.

21. Rémond D, Shahar DR, Gille D, et al. Understanding the gastrointestinal tract of the elderly to develop dietary solutions that prevent malnutrition. Oncotarget. 2015; 6(16): 13858-13898, doi: 10.18632/ oncotarget.4030, indexed in Pubmed: 26091351.

22. Patterson JE. The pre-travel medical evaluation: the traveler with chronic illness and the geriatric traveler. Yale J Biol Med. 1992; 65(4): 317-327, indexed in Pubmed: 1290273.

23. Driemeier M, de Oliveira PA, Druzian AF, et al. Late diagnosis: a factor associated with death from visceral leishmaniasis in elderly patients. Pathog Glob Health. 2015; 109(6): 283-289, doi: 10.1179/20477 73215Y.0000000029, indexed in Pubmed: 26257311. 
24. Gavazzi G, Krause KH. Ageing and infection. Lancet Infect Dis. 2002; 2(11): 659-666, indexed in Pubmed: 12409046.

25. Farage MA, Miller KW, Elsner $P$, et al. Characteristics of the Aging Skin. Adv Wound Care (New Rochelle). 2013; 2(1): 5-10, doi:10.1089/wound.2011.0356, indexed in Pubmed: 24527317.

26. McIntosh IB. Health hazards and the elderly traveler. J Travel Med. 1998; 5(1): 27-29, indexed in Pubmed: 9917179.

27. St-Onge MP, Gallagher D. Body composition changes with aging: the cause or the result of alterations in metabolic rate and macronutrient oxidation? Nutrition. 2010; 26(2): 152-155, doi: 10.1016/j. nut.2009.07.004, indexed in Pubmed: 20004080.

28. Masnoon N, Shakib S, Kalisch-Ellett L, et al. What is polypharmacy? A systematic review of definitions. BMC Geriatr. 2017; 17(1): 230, doi:10.1186/s12877-017-0621-2, indexed in Pubmed: 29017448.

29. Kim HA, Shin JY, Kim MH, et al. Prevalence and predictors of polypharmacy among Korean elderly. PLoS One. 2014; 9(6): e98043, doi:10.1371/journal.pone.0098043, indexed in Pubmed: 24915073.

30. Jyrkkä J, Enlund H, Korhonen MJ, et al. Polypharmacy status as an indicator of mortality in an elderly population. Drugs Aging. 2009; 26(12): 1039-1048, doi: 10.2165/11319530-00000000000000, indexed in Pubmed: 19929031.

31. Sabzwari SR, Qidwai W, Bhanji S. Polypharmacy in elderly: a cautious trail to tread. J Pak Med Assoc. 2013; 63(5): 624-627, indexed in Pubmed:23757993.

32. Maher RL, Hanlon J, Hajjar ER. Clinical consequences of polypharmacy in elderly. Expert Opin Drug Saf. 2014; 13(1): 57-65, doi: 10.1517/14740338.2013.827660, indexed in Pubmed: 24073682

33. Doan J, Zakrzewski-Jakubiak H, Roy J, et al. Prevalence and risk of potential cytochrome P450-mediated drug-drug interactions in older hospitalized patients with polypharmacy. Ann Pharmacother. 2013; 47(3): 324-332, doi: 10.1345/aph.1R621, indexed in Pubmed: 23482734.

34. World Health Organisation. Malaria. https://www.who.int/malaria/ en/ (Accessed: 10 Nov 2018).

35. Rees E, Saavedra-Campos M, Usdin M, et al. Trend analysis of imported malaria in London; observational study 2000 to 2014. Travel Med Infect Dis. 2017; 17: 35-42, doi: 10.1016/j.tmaid.2017.04.004, indexed in Pubmed: 28456684.

36. Allen N, Bergin C, Kennelly SP. Malaria in the returning older traveler. Trop Dis Travel Med Vaccines. 2016; 2: 2, doi: 10.1186/s40794016-0018-9, indexed in Pubmed: 28883946.

37. Centers for Disease Control and Prevention. Parasites - Malaria. https://www.cdc.gov/parasites/malaria/index.html (Accessed: 10 Nov 2018).

38. van Genderen PJJ, van Thiel PP, Mulder PGH, et al. Trends in the knowledge, attitudes and practices of travel risk groups towards prevention of malaria: results from the Dutch Schiphol Airport Survey 2002 to 2009. Malar J. 2012; 11: 179, doi: 10.1186/1475-287511-179, indexed in Pubmed:22642661.

39. Checkley AM, Smith A, Smith V, et al. Risk factors for mortality from imported falciparum malaria in the United Kingdom over 20 years: an observational study. BMJ. 2012; 344: e2116, doi: 10.1136/bmj. e2116, indexed in Pubmed: 22454091.

40. World Health Organisation. Dengue control. http://www.who.int/ denguecontrol/en/ (Accessed: 6 Nov 2018).

41. Goto K, Hatakeyama S, Okamoto K, et al. Dengue hemorrhagic fever in an adult traveler returning to Japan. Intern Med. 2012; 51(13): 1779-1782, indexed in Pubmed: 22790145.

42. Rowe EK, Leo YS, Wong JGX, et al. Challenges in dengue fever in the elderly: atypical presentation and risk of severe dengue and hospital-acquired infection [corrected]. PLoS Negl Trop Dis. 2014;
8(4): e2777, doi: 10.1371/journal.pntd.0002777, indexed in Pubmed: 24699282.

43. Stanaway JD, Shepard DS, Undurraga EA, et al. The global burden of dengue: an analysis from the Global Burden of Disease Study 2013. Lancet Infect Dis. 2016; 16(6): 712-723, doi: 10.1016/ S1473-3099(16)00026-8, indexed in Pubmed: 26874619.

44. Olivero RM, Hamer DH, MacLeod WB, et al. Dengue Virus Seroconversion in Travelers to Dengue-Endemic Areas. Am J Trop Med Hyg. 2016; 95(5): 1130-1136, doi: 10.4269/ajtmh.16-0159, indexed in Pubmed: 27573631

45. Hsieh CC, Cia CT, Lee JC, et al. A Cohort Study of Adult Patients with Severe Dengue in Taiwanese Intensive Care Units: The Elderly and APTT Prolongation Matter for Prognosis. PLoS Negl Trop Dis. 2017; 11(1): e0005270, doi: 10.1371/journal.pntd.0005270, indexed in Pubmed: 28060934.

46. Centers for Disease Control and Prevention. Parasites - American Trypanosomiasis (also known as Chagas Disease).https://www.cdc. gov/parasites/chagas/ (Accessed: 18 Nov 2018).

47. Pereira LS, Freitas EC, Fidalgo AS, et al. Clinical and epidemiological profile of elderly patients with Chagas disease followed between 2005-2013 by pharmaceutical care service in Ceará State, Northeastern Brazil. Rev Inst Med Trop Sao Paulo. 2015; 57(2): 145-152, doi: 10.1590/S0036-46652015000200008, indexed in Pubmed: 25923894

48. Alves RM, Thomaz RP, Almeida EA, et al. Chagas' disease and ageing: the coexistence of other chronic diseases with Chagas' disease in elderly patients. Rev Soc Bras Med Trop. 2009; 42(6): 622-628, indexed in Pubmed: 20209343.

49. Pereira MB, Batista AM, Aguiar C, et al. The detection of Trypanosoma cruzi by nested-PCR in elderly patients: relationship to the clinical and epidemiological profile. Pathog Glob Health. 2016; 110(6): 228-232, doi: 10.1080/20477724.2016.1232850, indexed in Pubmed: 27666187.

50. Centers for Disease Control and Prevention. Parasites - Leishmaniasis. https://www.cdc.gov/parasites/leishmaniasis/index. html (Accessed: 13 Nov 2018).

51. Gebremichael Tedla D, Bariagabr FH, Abreha HH. Incidence and Trends of Leishmaniasis and Its Risk Factors in Humera, Western Tigray. J Parasitol Res. 2018; 2018: 8463097, doi: 10.1155/2018/8463097, indexed in Pubmed: 30345109.

52. Montalvo AM, Fraga J, Blanco O, et al. Imported leishmaniasis cases in Cuba (2006-2016): what have we learned. Trop Dis Travel Med Vaccines. 2018; 4: 7, doi: 10.1186/s40794-018-0067-3, indexed in Pubmed: 30094054.

53. Leder K. Advising travellers about management of travellers' diarrhoea. Aust Fam Physician. 2015; 44(1-2): 34-37, indexed in Pubmed: 25688957.

54. Giddings SL, Stevens AM, Leung DT. Traveler's diarrhea. Med Clin North Am. 2016; 100(2): 317-330, doi: 10.1016/j. mcna.2015.08.017, indexed in Pubmed: 26900116.

55. Parise I. Travelling safely to places at high altitude - Understanding and preventing altitude illness. Aust Fam Physician. 2017; 46(6): 380-384, indexed in Pubmed: 28609593.

56. Hartman-Ksycińska A, Kluz-Zawadzka J, Lewandowski B. High altitude illness. Przegl Epidemiol. 2016; 70(3): 490-499, indexed in Pubmed: 27888818.

57. Luks AM, Swenson ER, Bärtsch P. Acute high-altitude sickness. Eur Respir Rev. 2017; 26(143): pii: 160096, doi:10.1183/16000617.00962016, indexed in Pubmed: 28143879.

58. Bazemore AW, Huntington M. The pretravel consultation. Am Fam Physician. 2009; 80(6): 583-590, indexed in Pubmed: 19817324.

59. Hamer DH, MacLeod WB, Chen LH, et al. Pretravel Health Preparation of International Travelers: Results From the Boston Area Travel Medicine 
Network. Mayo Clin Proc Innov Qual Outcomes. 2017; 1(1): 78-90, doi:10.1016/j.mayocpiqo.2017.04.001, indexed in Pubmed: 30225404.

60. McIntosh IB. The pre-travel health consultation. J Travel Med. 2015; 22(3): 143-144, doi: 10.1111/jtm.12182, indexed in Pubmed: 25904464.

61. Baggett HC, Graham S, Kozarsky PE, et al. Pretravel health preparation among US residents traveling to India to VFRs: importance of ethnicity in definingVFRs. J Travel Med. 2009; 16(2): 112-118, doi: 10.1111/j.17088305.2008.00284.x, indexed in Pubmed: 19335811.

62. Weinberger B, Grubeck-Loebenstein B. Vaccines for the elderly. Clin Microbiol Infect. 2012; 18 Suppl 5: 100-108, doi: 10.1111/j.14690691.2012.03944.x, indexed in Pubmed: 22862783.

63. Domnich A, Arata L, Amicizia D, et al. Effectiveness of MF59-adjuvanted seasonal influenza vaccine in the elderly: A systematic review and meta-analysis. Vaccine. 2017; 35(4): 513-520, doi: 10.1016/j. vaccine.2016.12.011, indexed in Pubmed: 28024956.

64. Mutsch M, Tavernini M, Marx A, et al. Influenza virus infection in travelers to tropical and subtropical countries. Clin Infect Dis. 2005; 40(9): 1282-1287, doi: 10.1086/429243, indexed in Pubmed: 15825030.

65 . Weinberger B. Vaccines for the elderly: current use and future challenges. Immun Ageing. 2018; 15: 3, doi: 10.1186/s12979017-0107-2, indexed in Pubmed: 29387135.

66. Falkenhorst G, Remschmidt C, Harder T, et al. Effectiveness of the 23-Valent Pneumococcal Polysaccharide Vaccine (PPV23) against Pneumococcal Disease in the Elderly: Systematic Review and Meta-Analysis. PLoS One. 2017; 12(1): e0169368, doi: 10.1371/ journal.pone.0169368, indexed in Pubmed: 28061505.

67. Lal H, Cunningham AL, Godeaux 0, et al. ZOE-50 Study Group. Efficacy of an adjuvanted herpes zoster subunit vaccine in older adults. N Engl J Med. 2015; 372(22): 2087-2096, doi: 10.1056/ NEJMoa1501184, indexed in Pubmed: 25916341.

68. Centers for Disease Control and Prevention. Yellow Fever Vaccine. https://www.cdc.gov/yellowfever/vaccine/index. html (Accessed: 23 Nov 2018).

69. Lawrence GL, Burgess MA, Kass RB. Age-related risk of adverse events following yellow fever vaccination in Australia. Commun Dis Intell Q Rep. 2004; 28(2): 244-248, indexed in Pubmed: 15460963.

70. Aasheim ET, Seymour M, Balogun K, et al. Acute hepatitis A in an elderly patient after care worker travel to high endemicity country. Hum Vaccin Immunother. 2013; 9(11): 2480-2482, indexed in Pubmed: 23880980.

71. Mutsch M, Spicher VM, Gut C, et al. Hepatitis A virus infections in travelers, 1988-2004. Clin Infect Dis. 2006; 42(4): 490-497, doi: 10.1086/499816, indexed in Pubmed: 16421793.

72. Stuurman AL, Marano C, Bunge EM, et al. Impact of universal mass vaccination with monovalent inactivated hepatitis $A$ vaccines: a systematic review. Hum Vaccin Immunother. 2017; 13(3): 724-736, doi: 10.1080/21645515.2016.1242539, indexed in Pubmed: 27786671.

73. Poovorawan K, Soonthornworasiri N, Sa-Angchai P, et al. Hepatitis B vaccination for international travelers to Asia. Trop Dis Travel Med Vaccines. 2016; 2: 14, doi: 10.1186/s40794-016-0031-z, indexed in Pubmed: 28883958.

74. Wong J, Payne M, Hollenberg S. A double-dose hepatitis B vaccination schedule in travelers presenting for late consultation. J Travel Med. 2014; 21(4): 260-265, doi: 10.1111/jtm.12123, indexed in Pubmed: 24796543.
75. Van Der Meeren O, Crasta P, Cheuvart B, et al. Characterization of an age-response relationship to GSK's recombinant hepatitis $B$ vaccine in healthy adults: An integrated analysis. Hum Vaccin Immunother. 2015; 11(7): 1726-1729, doi: 10.1080/21645515.2015.10397 58 , indexed in Pubmed:25996260.

76. Connor BA, Jacobs RJ, Meyerhoff AS. Hepatitis B risks and immunization coverage among American travelers. J Travel Med. 2006; 13(5): 273-280, doi: 10.1111/j.1708-8305.2006.00055.x, indexed in Pubmed: 16987126.

77. Mast EE, Weinbaum CM, Fiore AE, et al. A comprehensive immunization strategy to eliminate transmission of hepatitis $B$ virus infection in the United States: recommendations of the Advisory Committee on Immunization Practices (ACIP) Part II: immunization of adults. MMWR Recomm Rep. 2006; 55(RR-16): 1-33; quiz CE1, indexed in Pubmed: 17159833.

78. Smeti P, Pavli A, Katerelos P, et al. Typhoid vaccination for international travelers from Greece visiting developing countries. J Travel Med. 2014; 21(2): 99-103, doi: 10.1111/jtm.12076, indexed in Pubmed: 24251544.

79. Wagner KS, Freedman JL, Andrews NJ, et al. Effectiveness of the typhoid Vi vaccine in overseas travelers from England. J Travel Med. 2015; 22(2): 87-93, doi: 10.1111/jtm.12178, indexed in Pubmed: 25444695.

80. Mahon BE, Newton AE, Mintz ED. Effectiveness of typhoid vaccination in US travelers. Vaccine. 2014; 32(29): 3577-3579, doi: 10.1016/j.vaccine.2014.04.055, indexed in Pubmed: 24837780.

81. Gautret P, Parola P. Rabies vaccination for international travelers. Vaccine. 2012; 30(2): 126-133, doi: 10.1016/j.vaccine.2011.11.007, indexed in Pubmed: 22085557.

82. Mills DJ, Lau CL, Fearnley EJ, et al. The immunogenicity of a modified intradermal pre-exposure rabies vaccination schedule-a case series of 420 travelers. J Travel Med. 2011; 18(5): 327-332, doi: 10.1111/j.1708-8305.2011.00540.x, indexed in Pubmed: 21896096.

83. Hahn WO, Pottinger PS. Malaria in the traveler: how to manage before departure and evaluate upon return. Med Clin North Am. 2016; 100(2): 289-302, doi: 10.1016/j.mcna.2015.09.008, indexed in Pubmed: 26900114.

84. McKeage K, Scott L. Atovaquone/proguanil: a review of its use for the prophylaxis of Plasmodium falciparum malaria. Drugs. 2003; 63(6): 597-623, doi: 10.2165/00003495-200363060-00006, indexed in Pubmed: 12656656.

85. Warne B, Weld LH, Cramer JP, et al. EuroTravNet Network. Travel-related infection in European travelers, EuroTravNet 2011. J Travel Med. 2014; 21(4): 248-254, doi: 10.1111/jtm.12120, indexed in Pubmed: 24750378.

86. Hill DR. Health problems in a large cohort of Americans traveling to developing countries. J Travel Med. 2000; 7(5): 259-266, indexed in Pubmed: 11231210.

87. Wingate D, Phillips SF, Lewis SJ, et al. Guidelines for adults on self-medication for the treatment of acute diarrhoea. Aliment Pharmacol Ther. 2001; 15(6): 773-782, indexed in Pubmed: 11380315.

88. Michal Stevens A, Esposito DH, Stoney RJ, et al. Clostridium difficile infection in returning travellers. J Travel Med. 2017; 24(3), doi: 10.1093/jtm/taw099, indexed in Pubmed: 28355613.

89. Luks AM, Swenson ER, Bärtsch P. Acute high-altitude sickness. Eur Respir Rev. 2017; 26(143): pii:160096, doi: 10.1183/16000617.00962016, indexed in Pubmed: 28143879. 